\title{
DISTANCE-TRANSITIVE GRAPHS OF VALENCY FIVE
}

\author{
by A. GARDINER and CHERYL E. PRAEGER*
}

(Received 10th August 1985)

\section{Introduction}

If $u$ and $v$ are vertices of the (finite, connected) graph $\Gamma$, let $d(u, v)$ denote the length of the shortest path joining $u$ to $v$ in $\Gamma$. The graph $\Gamma$ is said to be distance-transitive if whenever $d(u, v)=d\left(u^{\prime}, v^{\prime}\right)$, there exists an automorphism $g$ of $\Gamma$ such that $u^{g}=u^{\prime}$ and $v^{g}=v^{\prime}$. Distance-transitive graphs of valency 3 and 4 were originally classified $[2,11,12$, 13] by using a computer to generate all "feasible intersection arrays" (cf. [1, Chapter 20]). In both cases a classification has since been given by hand $[4,5]$. We continue this latter tradition and prove the following theorem-which was recently proved independently by Ivanov et al. using a computer [10].

Theorem. $A$ distance-transitive graph of valency five is one of the following: $K_{6}$, $\left(2 . K_{6}\right)_{2},\left(2 . K_{6}\right)_{4}, H_{11}, Q_{5}, \square_{5}, K_{5,5}, 5 . K_{5,5}, O_{5}, 2 . O_{5}, 2 . \square_{5}, \Sigma_{6}, P_{3}(4), P_{4}(4)$.

$K_{6}$ is the complete graph, and $K_{5,5}$ the complete bipartite graph, of valency five. $Q_{5}$ is the 5-dimensional cube, and $\square_{5}$ is the antipodal quotient of $Q_{5} . O_{5}$ is the odd graph of valency five. $H_{11}$ is the incidence graph of points and blocks in the unique symmetric $2-(11,5,2)$ design. $\Sigma_{6}$ has as vertices the thirty-six subgroups of order 20 in $S_{6}$, two such being adjacent when they intersect in a subgroup of order $4\left(\left[1\right.\right.$, p. 153]). $P_{3}(4)$ and $P_{4}(4)$ are the incidence graphs respectively of the projective plane of order 4 and the classical generalised quadrangle of order $(4,4)$ associated with $P S p(4,4)$. If $\Delta$ is a graph of diameter $d$, then $r . \Delta$ denotes an $r$-fold antipodal covering of $\Delta$ with diameter $2 d$, whereas $(r . \Delta)_{y}$ denotes an $r$-fold antipodal covering of $\Delta$ with diameter $2 d+1$ in which the parameter $c_{d+1}=\gamma$. The antipodal covers occurring in the theorem are all unique. (For the definitions and basic properties of the parameters $a_{i}, b_{i}, c_{i}$ associated with the distance-transitive graph $\Gamma$ see $[1$, Chapter 20]. For information about antipodal coverings see $[6]$.)

\section{The two outstanding cases}

Given a group $G$ acting distance-transitively on the graph $\Gamma$ we introduce an extra parameter " $s$ ". An $s$-arc in $\Gamma$ is a sequence $(u(0), u(1), \ldots, u(s))$ of $s+1$ vertices, each adjacent to the next and such that $u(i-1) \neq u(i+1)(0<i<s)$. The group $G$ is $s$-arctransitive on $\Gamma$ if $G$ acts transitively on the set of $s$-arcs of $\Gamma$, but not on the set of $(s+1)$-arcs.

\footnotetext{
- This paper forms part of the Proceedings of the conference Groups-St Andrews 1985.
} 
Lemma 1. ([3, Lemma 2.4 and Proposition 3.5]). Let $\Gamma$ be a distance-transitive graph of valency 5 and girth $g$. Let $\{u, v\}$ be an edge of $\Gamma$. Then $G=$ Aut $\Gamma$ is s-arc-transitive on $\Gamma$ for some $s \geqq 1$ and one of the following holds:

(i) $g=3$ and $\Gamma=K_{6}$, or $\Gamma=\left(2 . K_{6}\right)_{2}$-the icosahedron;

(ii) $g=4, \Gamma=\left(2 . K_{6}\right)_{4}$, and $s=1$ or 2 ;

(iii) $g=4, s=2$, and $\Gamma=H_{11}, \Gamma=Q_{5}, \Gamma=\square_{5}$, or $\Gamma=K_{5,5}$;

(iv) $g=4, s=3$, and $\Gamma=K_{5,5}$;

(v) $g=5, s=2$, and either (a) $G_{1}(u)=1, G(u)=F_{5.4}, A_{5}$, or $S_{5}$, or (b) $G_{1}(u v)=$ $1 \neq G_{1}(u)=Z_{2}, G(u)=F_{5.4} \curlywedge Z_{4}=F_{5.4} \times Z_{2}$

(vi) $g=6, \Gamma=5 . K_{5.5}$, and $s=2$ or 3 ;

(vii) $g=6, s=3$, and $\Gamma=O_{5}$, or $\Gamma=2 . O_{5}$;

(viii) $g=6, s=4$, and $\Gamma=P_{3}(4)$;

(ix) $g=7, s=3$, and either (a) $G_{1}(u v)=1 \neq G_{1}(u)=Z_{4}, G(u)=F_{5.4} \times Z_{4}$, or (b) $G_{1}(u v)=$ $1 \neq G_{1}(u)=A_{4}$ or $S_{4}, G(u)=A_{5} \times A_{4}, S_{5} \curlywedge S_{4}$, or $S_{5} \times S_{4}$;

(x) $g \geqq 8, s \geqq 4$.

Here $G(u)$ denotes the stabiliser of the vertex $u$ in $G, G_{1}(u)$ denotes the pointwise stabiliser of $u$ and each of its neighbours, and $G_{1}(u v)=G_{1}(u) \cap G_{1}(v) . F_{5.4}$ is the Frobenius group of order 20.

Of the three outstanding cases (v), (ix), and (x) in Lemma 1, the last may appear the most intractable. The program proposed in [3] was based on the assumption that this case was likely to be the first to be resolved in complete generality (that is, for all valencies). This has recently been borne out by a result of Weiss [14], of which the following is a corollary.

Lemma 2. ([14]). Let $\Gamma$ be a distance-transitive graph of valency 5 and girth $\geqq 8$. If $\Gamma$ is $s$-arc transitive for some $s \geqq 4$, then $\Gamma=P_{4}(4)$.

The rest of this paper is devoted to the two remaining cases: (v) $g=5, s=2$, and (ix) $g=7, s=3$.

\section{Case (v)}

Case (v). $g=5, s=2$ and either (a) $G_{1}(u)=1, G(u)=F_{5.4}, A_{5}$, or $S_{5}$, or (b) $G_{1}(u v)=$ $1 \neq G_{1}(u)=Z_{2}, G(u)=F_{5.4} \times Z_{2}$.

We consider the possible values of the parameter $a_{2}$ in turn.

If $a_{2}=4$, then $\Gamma$ would be a Moore graph of valency 5 and diameter 2, contrary to [8]. Suppose $a_{2}=3$. Let $(u, v, w)$ be a 2 -arc in $\Gamma$. Then $G_{1}(u)=1$ (since $G_{1}(v) \triangleleft G(v w)$ acts $\frac{1}{2}$-transitively on $\Gamma(w)-\{v\}$ and has a fixed point, namely $\Gamma(w) \cap \Gamma_{3}(u)$; so $G_{1}(v)=$ $\left.G_{1}(w) \triangleleft\langle G(v), G(w)\rangle=G\right)$. Also $G(u) \neq F_{5.4}$ (otherwise $\left\langle\Gamma_{2}(u)\right\rangle$ would be a trivalent Cayley graph for $F_{5.4}$ with girth $\geqq 5$, which is impossible). Thus $G(u)=A_{5}$ or $\mathrm{S}_{5}$. Give $v$ the label 1, and label the other vertices of $\Gamma(u)$ with $2,3,4,5$. Then $G(u w)=G(u v w)=A_{3}$ or $S_{3}$, and so fixes exactly two points in $\Gamma(u)$, namely $v=1$ and one other-say 2 . So we 
may label each vertex $w \in \Gamma_{2}(u)$ with the ordered pair $(1,2)$ of points in $\Gamma(u)$ which are fixed by $G(u w)$, the first coordinate being precisely $\Gamma(u) \cap \Gamma(w) . G(u w)$ acts transitively on the three vertices of $\Gamma(w) \cap \Gamma_{2}(u)$, hence none of these vertices is joined to 1 or 2 (otherwise we would get a 3-gon or a 4-gon), none of them has 2 as second coordinate (or they all would, whence $\left\langle\Gamma_{2}(u)\right\rangle$ consists of five copies of $K_{4}$, so $\Gamma$ would contain a 3 -gon), and none of them has 1 as second coordinate (or they all would and $(1,2),(3,1)$, $(1,5),(4,1)$ would form a 4-gon in $\left.\left\langle\Gamma_{2}(u)\right\rangle\right)$. We may therefore assume that the three remaining vertices of $\Gamma(u)$ have been labelled in such a way that $(1,2)$ is joined to $(3,4)$, $(4,5),(5,3)$. It follows that $G(u w) \neq S_{3}$, so $G(u)=A_{5}$. But then $(3,4)$ is joined to $(1,2)$, $(2,5),(5,1)$, so $\left\langle\Gamma_{2}(u)\right\rangle=\left(2 . O_{3}\right)_{1}$-the dodecahedron. Thus the vertex 2 is in $\Gamma_{3}((1,2))$, and $\Gamma(2) \cap \Gamma_{2}((1,2)) \supseteqq\{u,(2,3),(2,4),(2,5)\}$ so $c_{3} \geqq 4$. Now $c_{3} \neq 5$ (otherwise $G(u)=A_{5}$ could not act transitively on the four points of $\Gamma_{3}(u)$ ). Hence $c_{3}=4$. But then $a_{3}=0$ (as $a_{3}=1$ with $k_{3}=5$ is impossible), so $b_{3}=1, c_{4}=5$, and $\Gamma=2 . \square_{5}$.

Suppose next that $a_{2}=2$. Let $(u, v, w)$ be a 2-arc. Then $G(u, w)$ leaves $\Gamma(w) \cap \Gamma_{2}(u)$ invariant, so $G(u) \neq A_{5}$ or $S_{5}$. Hence $G(u)=F_{5.4}$ or $F_{5.4} \times Z_{2}$, and $\left\langle\Gamma_{2}(u)\right\rangle=4 C_{5}$ or $2 C_{10}$ (since neither $F_{5.4}$ nor $F_{5.4} \times Z_{2}$ can act vertex transitively on $C_{20}$ ). Let $A=\Gamma(v) \cap \Gamma_{2}(u)$, $B=\Gamma(u)-\{v\}, C=\Gamma(B) \cap \Gamma(A)$ (where $\Gamma(B)$ consists of all vertices adjacent to some vertex in $B$, and similarly for $\Gamma(A)), D=\Gamma(A) \cap \Gamma_{3}(u), E=\left(\Gamma(B) \cap \Gamma_{2}(u)\right)-\Gamma(A)$. Now $|\Gamma(e) \cap B|=1$ for each $e \in E$ (since $c_{2}=1$ ). Also $E$ is not a union of connected components of $\left\langle\Gamma_{2}(u)\right\rangle$ (since $\left.5 \nmid|E|\right)$, so we must have $\Gamma(E) \cap C \neq \varnothing$. Thus since $E \subseteq \Gamma_{3}(v)$ and $B \cup C \subseteq \Gamma_{2}(v)$ we see that $c_{3} \geqq 2$. Moreover $|\Gamma(c) \cap E| \leqq 1$ for each $c \in C$, so $\Gamma(E) \cap E \neq \varnothing$ whence $a_{3} \geqq 1$ (since $E \subseteq \Gamma_{3}(v)$ ). Hence either $c_{3}=2$ or $c_{3}=4$. Suppose $c_{3}=4$. Then $a_{3}=1$. Hence $\Gamma$ has intersection array $\{5,4,2 ; 1,1,4\}$ and is primitive, so the group $G=$ Aut $\Gamma$ (of order 36.20 or 36.40 ) has a unique minimal normal subgroup $M$. Thus $M=A_{6}, \Gamma=\Sigma_{6}$, and Aut $\Gamma=$ Aut $S_{6}$.

Now suppose that $c_{3}=2$-still with $a_{2}=2, a_{3} \geqq 1$. Then $a_{3} \neq 3$ (otherwise $\Gamma$ would be primitive on 46 points, contradicting [15, Theorem 31.1]). Thus $a_{3}=2$ or $a_{3}=1$. We saw above that $\Gamma(E) \cap E$ and $\Gamma(E) \cap C$ are non-empty. Moreover interchanging $u$ and $v$ interchanges $A$ and $B, D$ and $E$, and leaves $C$ invariant, so $\Gamma(D) \cap D$ and $\Gamma(D) \cap C$ are non-empty. Thus if $G(u)=F_{5.4} \times Z_{2}$, then we have the partial intersection diagram of Fig. 1 (since $G(u v)$ is then transitive on $C, D, E)$. If $G(u)=F_{5.4}$, then $G(u v)$ has two orbits of length four on each of $C, D, E$ : if $\left\langle\Gamma_{2}(u)\right\rangle=4 C_{5}$, then the vertices of $A$ belong to distinct components and it is easy to see that the same partial intersection diagram applies; if $\left\langle\Gamma_{2}(u)\right\rangle=2 C_{10}$, then $A$ must consist of two "opposite pairs" of vertices-one from each of the two 10-gons-and it is not hard to see that this forces the same partial intersection diagram. If $a_{3}=1$ and $b_{3}=2$, then for each 3-arc $(u, v, w, x)$ with $u \in \Gamma_{3}(x), b_{3}=2$ implies that $\Gamma(u) \cap \Gamma_{4}(x)=\left\{v_{1}, v_{2}\right\}$. Thus if $\Gamma_{2}(x) \cap \Gamma(u)=\left\{v, v^{\prime}\right\}$ and $\Gamma\left(v^{\prime}\right) \cap \Gamma(x)=\left\{w^{\prime}\right\}$, then we can choose $z^{\prime} \in \Gamma\left(w^{\prime}\right) \cap \Gamma_{2}(u) \cap \Gamma(v)$ (since $w^{\prime} \in C$ ). Moreover $\left\{v_{1}, v_{2}\right\}=$ $\Gamma(u) \cap \Gamma_{3}(w)$ implies that $v^{\prime} \in \Gamma(u) \cap \Gamma_{2}(w)$ so we can choose $z \in \Gamma(w) \cap \Gamma_{2}(u) \cap \Gamma\left(v^{\prime}\right)$. But then the 3-arc $(u v w z)$ in $\left\langle\Gamma_{2}\left(w^{\prime}\right)\right\rangle$ has $\Gamma(u) \cap \Gamma\left(w^{\prime}\right)=\left\{v^{\prime}\right\}=\Gamma(z) \cap \Gamma\left(w^{\prime}\right)$, so the circuit containing $(u v w z)$ in $\left\langle\Gamma_{2}\left(w^{\prime}\right)\right\rangle$ cannot be "rotated" by an element of order 5 in $G\left(w^{\prime}\right)$. Thus $a_{3}=2, b_{3}=1$ and $c_{4} \geqq c_{3}=2$. Now $c_{4} \neq 5$ (otherwise $O_{5}(G(u)$ ) fixes each vertex $y \in \Gamma_{4}(u)$, so $O_{5}(G(u))=O_{5}(G(y)) \triangleleft\langle G(u), G(y)\rangle=G$ ). Also $c_{4} \neq 3$ (since $3 \nmid 20$ ). If $c_{4}=4$, then $a_{4} \neq 1$ (since $k_{4}=5$ is odd); so $a_{4}=0, b_{4}=1, c_{5}=5$, and $\Gamma$ would be a 2 -fold covering of a Moore graph of valency 5, contrary to [8]. Suppose $c_{4}=c_{3}=2$. Then $a_{4} \neq 3$ (or else $\left\langle\Gamma_{4}(u)\right\rangle$ is trivalent on ten vertices with girth $\geqq 5$, and so is isomorphic 


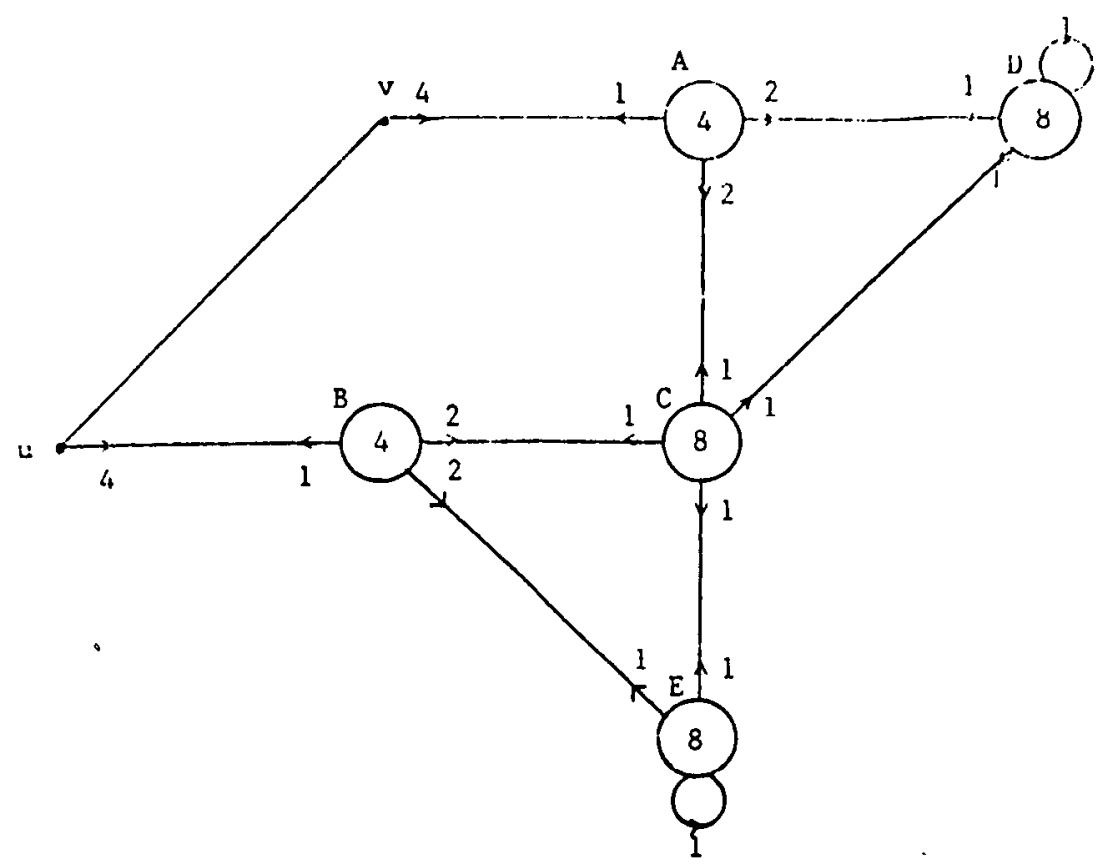

FIGURE 1

to the Petersen graph; but then for each $y \in \Gamma_{4}(u)$ we would have $C_{6}=$ $\left\langle\Gamma_{2}(y) \cap \Gamma_{4}(u)\right\rangle \leqq\left\langle\Gamma_{2}(y)\right\rangle=4 C_{5}$ or $\left.2 C_{10}\right)$. Hence $a_{4}=2, b_{4}=1$, so $c_{5}=5$ or 2 . Moreover $c_{5} \neq 5$ (or $\Gamma$ would be primitive on 58 points, contradicting [15, Theorem 31.1]). Thus $c_{5}=2$. Since $k_{5}=5$ is odd we cannot have $a_{5}=3$. Thus $a_{5}=2, b_{5}=1, c_{6}=5$. But then $\left\langle\Gamma_{5}(u) \cup \Gamma_{6}(u)\right\rangle$ contains a triangle. This completes the case $a_{2}=2$.

Suppose $a_{2}=1$. Then $b_{2}=3$, and $a_{3} \geqq 1$ (since $\left\langle\Gamma_{3}(v) \cap \Gamma_{2}(u)\right\rangle=6 K_{2}$ ). Hence $c_{3} \leqq 4$. Moreover $c_{3} \neq 4$ (since if $c_{3}=4$ then $a_{3}=1$ and $\Gamma$ would be primitive on 41 vertices, contradicting [9, Satz 21.3]). Suppose $c_{3}=3$. Then $a_{3}=1$ and $b_{3}=1$ (otherwise $a_{3}=2$ and $\Gamma$ would be primitive on 46 vertices, contrary to [15, Theorem 31.1]). Hence $c_{4} \neq 5$ (otherwise $k_{4}=4$ and either $G(u) \geqq A_{5}$ could not act transitively on $\Gamma_{4}(u)$, or $G(u)$ is soluble and $O_{5}(G(u))$ fixes each $y \in \Gamma_{4}(u)$, so $\left.O_{5}(G(u))=O_{5}(G(y)) \triangleleft\langle G(u), G(y)\rangle=G\right)$. Thus $c_{4}=4, k_{4}=5$ is odd, so $a_{4} \neq 1$; hence $a_{4}=0, b_{4}=1, c_{5}=5$, and $\Gamma$ would be a 2 -fold cover of a Moore graph of valency 5, contradicting [8]. Suppose $c_{3}=2$. Then $k_{3}=30$, so $G(u)=A_{5}$ or $S_{5}$. If we label the vertices of $\Gamma(u)$ with the symbols $1,2,3,4,5$, each vertex $w$ of $\Gamma_{2}(u)$ receives a natural label $(i, j)$ where $\Gamma(w) \cap \Gamma(u)=\{i\}$ and $G(u w)$ fixes $i, j \in \Gamma(u)$. Since $a_{2}=1$ the vertex $(i, j)$ must be joined to the vertex $(j, i)$. Let $v=1, w=(1,2)$, $x \in \Gamma(w) \cap \Gamma_{3}(u)$. Then $G(u x)$ interchanges the two vertices $(1,2),(i, j) \in \Gamma(x) \cap \Gamma_{2}(u)$. Now $i \neq 1$ (since $\Gamma$ contains no 4-gons), and $i \neq 2$ (otherwise either $j=1$ so $\Gamma$ contains a 3-gon, or $j \neq 1$ and the 2 -arc $((1,2),(2,1), 2)$ lies in more than one 5-gon). Similarly $j \neq 1$. Thus either (i) $\{1,2\} \cap\{i, j\}=\varnothing$, or (ii) $j=2$.

(i) Suppose $\{1,2\} \cap\{i, j\}=\varnothing$. Then $k_{3}=30$, so $G(u)$ must have an orbit of length 30 on the sixty pairs $\{(i, j),(k, l)\}$ with $\{i, j\} \cap\{k, l\}=\varnothing$. Hence $G(u)=A_{5}$. We may assume that $\Gamma(x) \cap \Gamma_{2}(u)=\{(1,2),(3,4)\}$ and give $x$ the label $\{(1,2),(3,4)\} . G(u x)=\langle(13)(24)\rangle$ has 
precisely two fixed points in $\Gamma_{3}(u)$, namely $x=\{(1,2),(3,4)\}$ and $x^{\prime}=\{(2,1),(4,3)\}$. Thus $a_{3}=2$ (otherwise $x$ would have to be joined to $x^{\prime}$ and we would get the 4-gon $\left(x, x^{\prime}\right.$, $(2,1),(1,2)))$. Let $A=\Gamma(v) \cap \Gamma_{2}(u), D=\Gamma_{2}(v) \cap \Gamma_{3}(u)=\Gamma(A) \cap \Gamma_{3}(u)$. Since $a_{2}=1$ in $\Gamma_{2}(v)$ we must have $\langle D\rangle=6 K_{2}$. Thus the two vertices in $\Gamma(x) \cap \Gamma_{3}(u)$ are of the form $\{(1, i),(j, k)\}$ and (applying $G(u x)=\langle(13)(24)\rangle)\left\{\left(3, i^{\prime}\right),\left(j^{\prime}, k^{\prime}\right)\right\}$. Using this and the fact that $\Gamma(x) \cap \Gamma_{3}(u)$ must be a self-paired suborbit of $G(u)^{\Gamma_{3}(u)}$ we get just five possibilities for $\Gamma(x) \cap \Gamma_{3}(u)=\left\{y, y^{\prime}\right\}$ say: (a) $y=\{(3,5),(1,4)\}, y^{\prime}=\{(1,5),(3,2)\} ;$ (b) $y=\{(5,4),(3,2)\}$, $y^{\prime}=\{(5,2),(1,4)\} ; \quad$ (c) $y=\{(1,4),(5,2)\}, \quad y^{\prime}=\{(3,2),(5,4)\} ; \quad$ (d) $y=\{(2,1),(3,5)\}, \quad y^{\prime}=$ $\{(4,3),(1,5)\}$; (e) $y=\{(4,5),(3,1)\}, y^{\prime}=\{(2,5),(1,3)\}$. In cases (a)-(c) we get $y^{\prime} \in \Gamma(y)$, so $\Gamma$ has girth 3 ; in case (d) we get a 4 -gon $((1,2), x, y,(2,1))$; in case (e) the 2-arc $\left(y, x, y^{\prime}\right)$ lies in two 5 -gons-one in $\left\langle\Gamma_{3}(u)\right\rangle$, the other being $\left(y, x, y^{\prime},(1,3),(3,1)\right)$. Thus case (i) does not occur.

(ii) Suppose now that $j=2$ : that is, if $x \in \Gamma(1,2) \cap \Gamma_{3}(u)$, then we may assume that $\Gamma(x) \cap \Gamma_{2}(u)=\{(1,2),(3,2)\}$. Then each vertex $x$ in $\Gamma_{3}(u)$ receives a natural label $x=$ $(2,(13))$ whose second coordinate is an unordered pair, or transposition, not involving the first coordinate. The 2-arc $(1,(1,2),(2,(13)))$ lies in a unique 5 -gon, which we may take to be $(1,(1,2),(2,(13)),(5,(14)),(1,5))$. Thus $G(u)=A_{5}$ (otherwise $G(u x)=\langle(13),(45)\rangle$ and the $G(u x)$-orbit containing $(5,(14))$ would have size four, contradicting $a_{3} \leqq 3$ ). Hence $G(u x)=\langle(13)(45)\rangle$, so $(2,(13))$ is also joined to $(4,(35))$, whence $a_{3} \geqq 2$. The $G(u)$ images of the edge $\{(2,(13)),(5,(14))\}$ form six 5 -gons in $\left\langle\Gamma_{3}(u)\right\rangle$. But then we get two 5-gons $\left((1,(52)),(3,(24)),(5,(14)), x^{\prime}, y^{\prime}\right)$ containing a single 2 -arc-one with $x^{\prime}=(2,(13))$, $y^{\prime}=(4,(35))$, and the other with $x^{\prime}=(1,5), y^{\prime}=(5,1)$-contradicting $a_{2}=1$. Thus case (ii) does not occur. Hence $c_{3} \neq 2$.

Suppose $c_{3}=1$. Then $k_{3}=60$, so $G(u)=A_{5}$ or $S_{5}$. As before $\left\langle\Gamma_{2}(v) \cap \Gamma_{3}(u)\right\rangle=6 K_{2}$, so $a_{3} \geqq 1, b_{3} \leqq 3$. Each edge $\left(w, w^{\prime}\right)$ in $\left\langle\Gamma_{2}(u)\right\rangle$ lies in exactly three 2 -arcs $\left(w, w^{\prime}, x^{\prime}\right)$ with $x^{\prime} \in \Gamma_{3}(u)$. And each such 2-arc lies in a unique 5-gon $\left(w, w^{\prime}, x^{\prime}, x^{\prime \prime}, x\right)$. Since $G(u)$ acts transitively on such 2-arcs, it must act transitively on the 30 corresponding 5-gons. Hence the possible vertices $x^{\prime \prime}$ opposite the edge $\left(w, w^{\prime}\right)$ must form a single $G(u)$-orbit whose length divides 30 . Thus $x^{\prime \prime} \in \Gamma_{4}(u), k_{4}$ divides 30 , and $c_{4}=\left|\Gamma\left(x^{\prime \prime}\right) \cap \Gamma_{3}(u)\right| \geqq\left|\left\{x^{\prime}, x\right\}\right|=2$. So either (a) $k_{4}=30$, and $b_{3}=2, c_{4}=4$ or $b_{3}=1, c_{4}=2$, or (b) $k_{4}=15$ and $b_{3}=1, c_{4}=4$. Each 5-gon $\left(u, v, w, w^{\prime}, v^{\prime}\right)$ in $\Gamma$ has stabiliser $A_{3}$ or $S_{3}$, and so is fixed by a unique subgroup $\langle h\rangle$ of order three. There is an element $g \in G$ of order five which "rotates" the 5 -gon and which normalises, and hence centralises, the subgroup $\langle h\rangle$. In both case (a) and case (b) we have $k_{0}=1$ and $k_{1} \equiv k_{2} \equiv k_{3} \equiv k_{4} \equiv 0(\bmod 5)$. Moreover $k_{i} \neq 0(\bmod 5)$ only if $c_{i}=5$, whence $i=d$. Thus since $G(u)=A_{5}$ or $S_{5}$ acts transitively on each $\Gamma_{i}(u)$ we have $k_{i} \geqq 5$ for each $i \geqq 5$. (The apparent possibilities $k_{d}=1$ or 2 would force $\Gamma$ to be antipodal, and this is not possible if $k_{4} \mid 30$.) It follows that $\sum_{i=0}^{d} k_{i}=|G: G(u)| \neq 0(\bmod 5)$. Thus the element $g$ must fix some vertex $z$. Since $G(z)=A_{5}$ or $S_{5}$, the element $h$ cannot fix $z$, so $h$ acts semiregularly on the fixed points of $g$ : in particular, $g$ fixes at least three points. But if $g$ fixes the vertex $z^{\prime} \in \Gamma_{j}(z)$ for some first $j \geqq 1$, then $\langle g\rangle$ must act transitively on $\Gamma\left(z^{\prime}\right)$, so $c_{j}=5, j=d$. Since $k_{d} \neq 2,3,4$, the only possibility in case (a) or (b) above is that $k_{4}=30, b_{4}=1, c_{5}=5, k_{5}=6$. But then $g$ fixes only $1+0+0+0+0+1$ points, which is a contradiction. This completes the case $a_{2}=1, c_{3}=1$, and hence completes case (v). 


\section{Case (ix)}

Case (ix). girth 7, s=3, $G_{1}(u v)=1$ and either (a) $G_{1}(u)=Z_{4}, G(u)=F_{5.4} \times Z_{4}$, or (b) $G_{1}(u)=A_{4}$ or $S_{4}, G(u)=A_{5} \times A_{4}, S_{5} \wedge S_{4}$, or $S_{5} \times S_{4}$.

Suppose first that $G(u) \triangleright A_{5} \times A_{4}$. If $(0,1,2,3)$ is a 3 -arc in $\Gamma$, the stabiliser $H=$ $G(0123) \geqq A_{3} \times A_{3}$ fixes a unique circuit $C=(0,1,2,3, \ldots, t-1)$ containing the 3 -arc $(0,1,2,3)$. Moreover for each $i, O_{3}\left(G_{1}(i) \cap H\right)=A_{3}$ fixes $\Gamma(i+2 k)(\bmod t)$ pointwise and acts non-trivially on each $\Gamma(i+2 k-1)(\bmod t)$; $($ compare [5, Lemma 3$]$ or [7, Lemmas 5.2-5.4]). Hence in particular $t$ must be even, so $t \geqq 8, a_{3}=3, b_{3}=1$. Let $\Gamma(3) \cap \Gamma_{3}(0)=$ $\left\{x_{1}, x_{2}, x_{3}\right\}$, and let $\Gamma_{2}\left(x_{i}\right) \cap \Gamma(0)=\left\{y_{i}\right\}(i=1,2,3)$. Then $O_{3}\left(G_{1}(0) \cap H\right)$ must permute the $x_{i}$ cyclically while fixing the $y_{i}$ pointwise, which is impossible. Thus we may assume that $G(u)=F_{5.4} \times Z_{4}$.

$\Gamma$ has girth 7 so $a_{3} \geqq 1, b_{3} \leqq 3$. If $a_{3}=4$, then $\Gamma$ would be a Moore graph of diameter 3 -contradicting [8]. If $a_{3}=3$, then $\left\langle\Gamma_{3}(u)\right\rangle$ has girth $\geqq 7$ and so must consist of two components of size 40 (otherwise $\left\langle\Gamma_{3}(u)\right\rangle$ would be a Cayley graph for $F_{5.4} \times Z_{4}$, whereas this group cannot be generated either by three involutions, or by a single element of order $\geqq 7$ together with an involution). But each component would then be a Cayley graph of girth $\geqq 7$ for some subgroup $H$ of index 2 in $G(u)$. However $H=F_{5.4} \times Z_{2}$ and $H=F_{5.4} \curlywedge Z_{4}$ cannot be generated either by three involutions, or by an element of order $\geqq 7$ and an involution; and though $H=D_{10} \times Z_{4}$ can be so generated (namely by an element $g$ of order 20 and an involution $t$ ), the corresponding Cayley graph has girth 6 (since $t$ inverts $g^{4}$ and centralises $g^{5}$, so $g t g=g^{-1} t g^{-1}$ ). Suppose $a_{3}=2$. Then $b_{3}=2$, so $c_{4}=2$ or 4 (since $k_{4}$ must divide $|G(u)|=80$ ). If $c_{4}=4$ then either (a) $a_{4}=1$ and $\Gamma$ is primitive on 2.73 points, contradicting [15, Theorem 31.1$]$, or (b) $a_{4}=0$, $b_{4}=1$, in which case we must have $c_{5}=4, a_{5}=1$ (since $c_{5}=5$ implies $5 \Varangle k_{5}$, whence for $\left.z \in \Gamma_{5}(u), O_{5}(G(u))=O_{5}(G(z)) \triangleleft\langle G(u), G(z)\rangle=G\right)$. But then if $v \in \Gamma(u),\left\langle\Gamma_{5}(u)\right\rangle=5 K_{2}$ and $\left|\Gamma_{4}(v) \cap \Gamma_{5}(u)\right|=8$, so $\left\langle\Gamma_{4}(v)\right\rangle$ must contain an edge, contradicting $a_{4}=0$. Thus we may assume that $c_{4}=2$. Clearly $a_{4} \neq 3$ (for the same reasons as $a_{3} \neq 3$ ). If $a_{4}=2$, then $\left\langle\Gamma_{4}(u)\right\rangle$ is a union of circuits of length $\geqq 7$, so $\left\langle\Gamma_{4}(u)\right\rangle=4 C_{20}$ or $8 C_{10}$ (since $F_{5.4} \times Z_{4}$ cannot act regularly on $C_{80}, 2 C_{40}, 5 C_{16}$, or $\left.10 C_{8}\right)$. Let $y \in \Gamma_{4}(u), y_{1}, y_{2} \in \Gamma(y) \cap \Gamma_{4}(u),\{z\}=$ $\Gamma(y) \cap \Gamma_{5}(u)$. Then $b_{4}=1$, so $\Gamma(u) \cap \Gamma_{5}(y)=\left\{v^{*}\right\}$ say. Now $b_{5} \leqq b_{4}=1$. If $c_{5}=2$ then $\Gamma(y) \cap \Gamma_{4}\left(v^{*}\right)=\Gamma(y) \cap \Gamma_{3}(u)$, and either $d>5, \quad b_{5}=1, \quad a_{5}=2, \quad\{z\}=\Gamma(y) \cap \Gamma_{6}\left(v^{*}\right)$, so $\Gamma(y) \cap \Gamma_{4}(u) \subseteq \Gamma(y) \cap \Gamma_{5}\left(v^{*}\right)$, or $d=5, a_{5}=3$, and again $\Gamma(y) \cap \Gamma_{4}(u) \subseteq \Gamma(y) \cap \Gamma_{5}\left(v^{*}\right)$. Thus the component of $\left\langle\Gamma_{4}(u)\right\rangle$ containing $y$ lies in $\Gamma_{5}\left(v^{*}\right)$. Since $G(u)$ acts transitively on $\Gamma(u)$, the number of components of $\left\langle\Gamma_{4}(u)\right\rangle$ should be divisible by $|\Gamma(u)|=5$, a contradiction. Hence $c_{5}=4$ (since $c_{5}=5$ would imply that $O_{5}(G(u))=$ $O_{5}(G(z)) \triangleleft\langle G(u), G(z)\rangle=G$ for each $z \in \Gamma_{5}(u)$ ). Moreover $a_{5}=0$ (otherwise $\Gamma$ would be primitive on 2.103 points, contradicting [15, Theorem 31.1]). If $c_{6}=5$, then $\Gamma$ would be a 5-fold cover of $P_{3}(4)$, contrary to [6]. Thus $c_{6}=4, a_{6}=0$ (otherwise $\Gamma$ would be primitive on 211 points contradicting [9, Satz 21.3]), $b_{6}=1, c_{7}=5$ and $\Gamma$ is a 2 -fold cover of a Moore graph of diameter 3, contrary to [8]. Thus $a_{4} \neq 2$.

Let $v \in \Gamma(u), A=\Gamma(u) \cap \Gamma_{2}(v), B=\Gamma(v) \cap \Gamma_{2}(u), C=\Gamma_{3}(v) \cap \Gamma_{2}(u), D=\Gamma_{3}(u) \cap \Gamma_{2}(v), E=$ $\Gamma_{4}(v) \cap \Gamma_{3}(u), F=\Gamma_{4}(u) \cap \Gamma_{3}(v), H=\Gamma_{3}(u) \cap \Gamma_{3}(v)$. Then $A, B, C, D$ are all $G(u v)$-orbits. Since $G(u v)$ acts semi-regularly on $\Gamma_{3}(u)$, both $E=E_{1} \cup E_{2}$ and $H=H_{1} \cup H_{2}$ break up into two $G(u v)$-orbits. Interchanging $u$ and $v$ we see that $F=F_{1} \cup F_{2}$ also consists of two $G(u v)$-orbits. Since $a_{3}=2$ and $G(u)=F_{5.4} \times Z_{4}$ acts regularly on $\Gamma_{3}(u)$, we have $\left\langle\Gamma_{3}(u)\right\rangle=$ 
$4 C_{20}$ or $8 C_{10}$. Since $D \subseteq \Gamma_{2}(v)$ and $a_{2}=0$, we have $\Gamma(D) \cap \Gamma_{3}(u) \subseteq H$. Moreover any union of components in $\left\langle\Gamma_{3}(u)\right\rangle$ must have size divisible by 10 , so $\Gamma(D) \cap H_{i} \neq \varnothing$ $(i=1,2)$. If $\Gamma(H) \cap H \neq \varnothing$, then since $D \cup H$ cannot be a union of components we must have one of the $G(u v)$-orbits on $H$, say $\left\langle H_{1}\right\rangle=8 K_{2}, \Gamma\left(H_{2}\right) \cap E=E_{2}$ (say), $\Gamma\left(E_{2}\right) \cap E=E_{1}$, $\left\langle E_{1}\right\rangle=8 K_{2}$. But then $|\Gamma(x) \cap E|=2$ for each $x \in E$, which is impossible since $E \leqq \Gamma_{4}(v)$ and $a_{4} \leqq 1$. Thus $\Gamma(H) \cap H=\varnothing, \Gamma(H) \cap E_{i} \neq \varnothing(i=1,2)$, and we have the partial intersection diagram in Fig. 2. In particular $a_{4} \geqq 1$, so $a_{4}=1$.

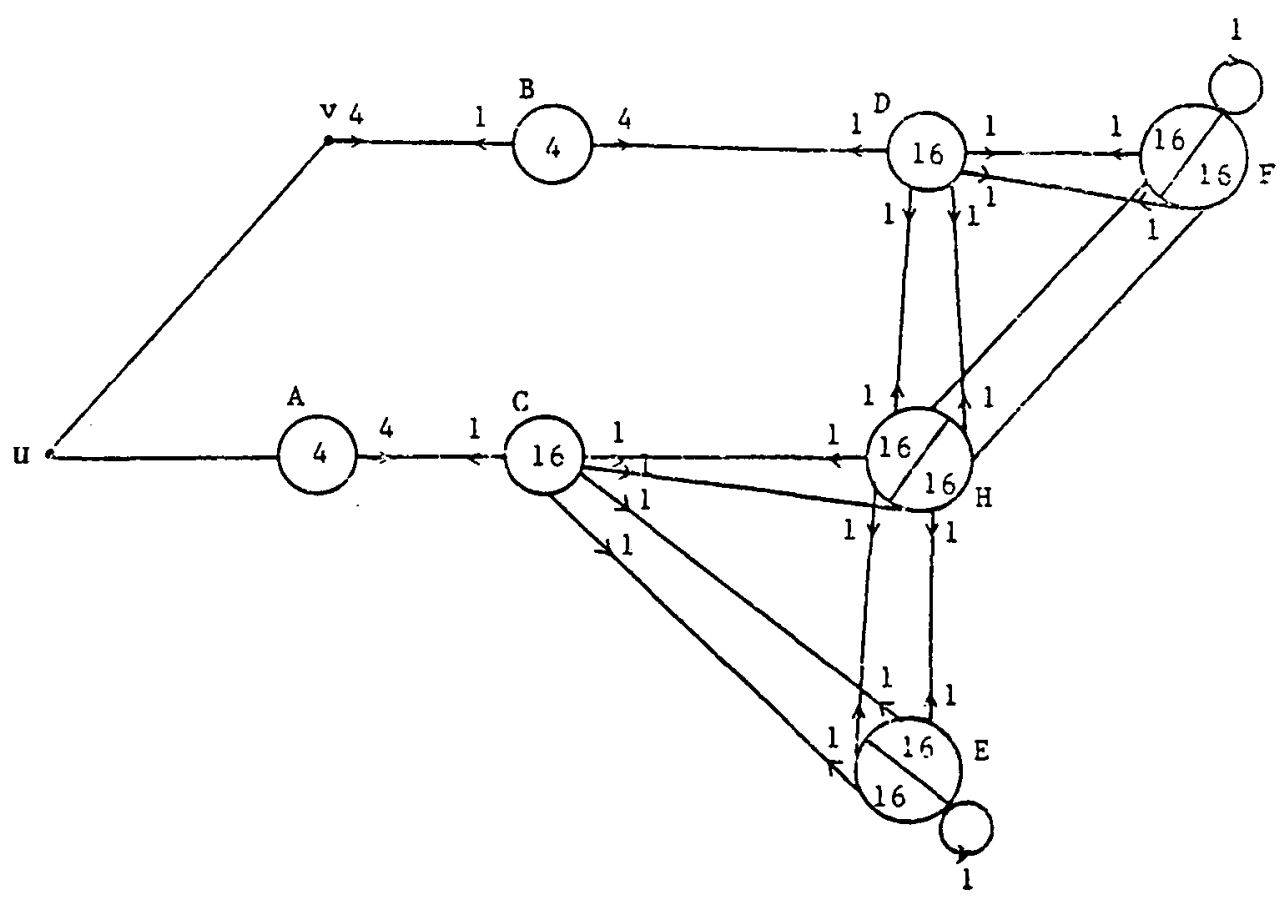

FIGURE 2

Suppose $\Gamma\left(E_{i}\right) \cap E_{i} \neq \varnothing(i=1,2)$. Then $\Gamma\left(F_{i}\right) \cap F_{i} \neq \varnothing(i=1,2)$. Let $\left(y_{1}, y_{2}\right)$ be an edge in $\left\langle F_{1}\right\rangle$. Then some element $g \in G(u v)=G_{1}(u) \times G_{1}(v)$ maps $y_{1}$ to $y_{2}$, and so inverts the edge $\left(y_{1}, y_{2}\right)$. Hence $g^{2}=1$. Let $\Gamma_{3}\left(y_{1}\right) \cap \Gamma(u)=\left\{v, v^{\prime}\right\}$. Then $y_{1} \in \Gamma_{3}\left(v^{\prime}\right)$, so $\left|\Gamma\left(y_{1}\right) \cap \Gamma_{3}\left(v^{\prime}\right)\right|=2$ (since $a_{3}=2$ ). Now $b_{4}=b_{3}=2$ (since $a_{4}=1$ ), so $\Gamma\left(y_{1}\right) \cap \Gamma_{5}(u)=\Gamma\left(y_{1}\right) \cap \Gamma_{4}\left(v^{\prime}\right)$. Thus $y_{2} \in \Gamma_{3}\left(v^{\prime}\right)$ and $\left\{v, v^{\prime}\right\}=\Gamma_{3}\left(y_{2}\right) \cap \Gamma(u)$. Thus $g$ fixes $v^{\prime}$. If $t_{u}, t_{v}$ denote the involutions in $G_{1}(u), G_{1}(v)$ respectively, then $g=t_{u}$ (since $t_{v}$ and $t_{u} t_{v}$ have only one fixed point in $\Gamma(u)$ ). But then $\langle g\rangle \triangleleft G(u)$, so the $\langle g\rangle$-orbits in $\Gamma_{4}(u)$ form a block system for the action of $G(u)$ on $\Gamma_{4}(u)$. Hence $g$ inverts every edge in $\Gamma_{4}(u)$. If $\left\{w_{i}\right\}=\Gamma\left(y_{i}\right) \cap \Gamma_{2}\left(v^{\prime}\right)$, and $\left\{y_{i}^{\prime}\right\}=$ $\left(\Gamma\left(w_{i}\right) \cap \Gamma_{4}(u)\right)-\left\{y_{i}\right\}(i=1,2)$, then $g$ must interchange $y_{1}^{\prime}$ and $y_{2}^{\prime}$, so $\left(y_{1}^{\prime}, y_{2}^{\prime}\right)$ must be an edge. But then $\left(y_{1}, y_{2}, w_{2}, y_{2}^{\prime}, y_{1}^{\prime}, w_{1}\right)$ would be a 6 -gon in $\Gamma$-a contradiction. Hence $\Gamma\left(F_{i}\right) \cap F_{i}=\varnothing=\Gamma\left(E_{i}\right) \cap E_{i}(i=1,2)$, and each circuit in $\left\langle\Gamma_{3}(u)\right\rangle$ runs through the five $G(u v)$-orbits $D, H_{1}, E_{1}, E_{2}, H_{2}$ in turn. Let $\left(y_{1}, y_{2}\right)$ be an edge in $\langle F\rangle$ with $y_{i} \in F_{i}(i=1,2)$. As before we have $\Gamma_{3}\left(y_{i}\right) \cap \Gamma(u)=\left\{v, v^{\prime}\right\}$, and let $\left\{w_{i}\right\}=\Gamma\left(y_{i}\right) \cap \Gamma_{2}\left(v^{\prime}\right)(i=1,2) . G_{1}(u)$ has four orbits $F_{i 1}, F_{i 2}, F_{i 3}, F_{i 4}$ of length 4 on each $F_{i}(i=1,2)$, which we may assume to be 
labelled such that $y_{1} \in F_{11}$ and $y_{2} \in F_{21}$, four orbits $H_{i 1}, H_{i 2}, H_{i 3}, H_{i 4}$ of length 4 on each $H_{i}$, labelled such that $w_{1} \in H_{11}$ and $w_{2} \in H_{21}$, and four orbits $C_{1}, C_{2}, C_{3}, C_{4}$ of length 4 on $C$, labelled such that $C_{1}=\Gamma\left(v^{\prime}\right)$. Moreover $\left(\Gamma_{2}\left(y_{i}\right) \cap \Gamma_{2}(u)\right)-\Gamma(v) \subseteq \Gamma\left(v^{\prime}\right)=C_{1}$, so $\Gamma_{2}\left(y_{i}^{*}\right) \cap \Gamma_{2}(u) \subseteq C_{1}$ for each $y_{i}^{*} \in F_{i 1}(i=1,2)$. It follows that each circuit in $\left\langle\Gamma_{3}(v)\right\rangle$ runs through five $G_{1}(u)$-orbits-say $F_{11}, F_{21}, H_{21}, C_{1}, H_{11}$-in turn. Thus the $G_{1}(u)$ orbits in $\Gamma_{3}(v)$ form a block system for the action of $G(v)$, with quotient $4 C_{5}$. If $x \in \Gamma_{3}(v)$, let $C(x)$ be the cycle in $\left\langle\Gamma_{3}(v)\right\rangle$ containing the vertex $x$. Since $G(v)$ acts regularly on $\Gamma_{3}(v)$ the element $g^{\prime} \in G(v)$ which maps $y_{1}$ (say) to the vertex opposite $y_{1}$ on $C\left(y_{1}\right)$ must belong to $G_{1}(u)$, and so induces a half turn on $C\left(y_{1}\right)$. But then $g^{\prime}=t_{u}$ (since $g^{\prime 2}=1$ ). Hence $t_{u}$ commutes with $O_{5}(G(v))$, whereas $t_{u}$ should invert $O_{5}(G(v))$.

Thus $a_{3}=1$ and $\left\langle\Gamma_{3}(u)\right\rangle=40 K_{2}$. Since 3 does not divide $|G(u)|, c_{4}=3$ and $k_{4}=80$. If $a_{4}=2$, then $G$ is primitive with order $186.80=2^{5} .3 .5 .31$. There are 186 Sylow 5subgroups (the Sylow 5-normalisers being precisely the vertex stabilisers). By Sylow's theorem there must be 32 Sylow 31-normalisers, each containing 31 Sylow 5-subgroups. Thus each Sylow 5-subgroup occurs in 32.31/186 Sylow 31-normalisers-a contradiction. Hence $a_{4} \leqq 1$. Let $v \in \Gamma(u), A=\Gamma(u) \cap \Gamma_{2}(v), B=\Gamma(v) \cap \Gamma_{2}(u), C=\Gamma_{3}(v) \cap \Gamma_{2}(u)$, $D=\Gamma_{3}(u) \cap \Gamma_{2}(v), \quad E=\Gamma_{4}(v) \cap \Gamma_{3}(u), \quad F=\Gamma_{4}(u) \cap \Gamma_{3}(v), \quad$ and $H=\Gamma_{3}(u) \cap \Gamma_{3}(v)$. Then $A, B, C, D, H$ are all $G(u v)$-orbits. Since $a_{3}=1$ we have for each $y \in D, \Gamma(y) \cap H \neq \varnothing$, and so for each $y \in E$ we must have $\Gamma(y) \cap E \neq \varnothing$. An element interchanging $u$ and $v$ would interchange $E$ and $F$ and leave $H$ invariant. Hence for each $y \in F$ we have $\Gamma(y) \cap F \neq \varnothing$ and $\Gamma(y) \cap H=\varnothing$. It follows that $a_{4}=1$ and we get the partial intersection diagram in Fig. 3. Thus $b_{4}=1$ and $c_{5} \geqq 4$ (since 3 does not divide 80). If $c_{5}=5$, then $G$ is primitive of degree $202=2.101$ contradicting [15, Theorem 31.1]. Thus $c_{5}=4$. If $a_{5}=1$, then $G$ is primitive of degree $206=2.103$, again contradicting [15, Theorem 31.1]. So $a_{5}=0, b_{5}=1$. Suppose $c_{6}=5$. Then $\Gamma$ is not antipodal (since $a_{4} \neq a_{2}$ ), so $G$ is primitive. But then for each $z \in \Gamma_{6}(u), O_{5}(G(u)) \triangleleft\langle G(u), G(z)\rangle=G$, a contradiction. Thus $c_{6}=4, a_{6}=0, b_{6}=1$, $c_{7}=5$ and $\Gamma$ is a 2-fold antipodal covering of a Moore graph of diameter 3 , contradicting [8]. This completes case (ix) and hence proves the theorem.

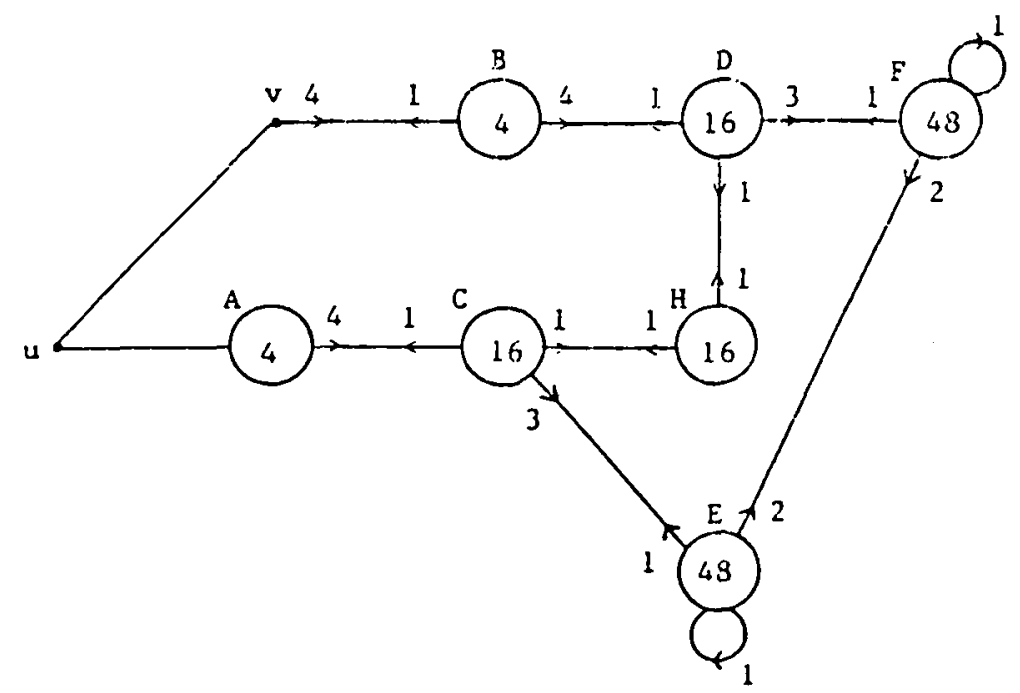

FIGURE 3 


\section{REFERENCES}

1. N. L. Biggs, Algebraic Graph Theory (Cambridge University Press, 1974).

2. N. L. Biggs and D. H. Smirh, On trivalent graphs, Bull. London Math. Soc. 3 (1971), 155158.

3. A. Gardiner, Classifying distance-transitive graphs, in Combinatorial Mathematics IX, Eds. E. J. Billington, S. Oates-Williams and A. P. Street (Springer Lecture Notes in Mathematics 952, 1982), 67-88.

4. A. Gardiner, On trivalent graphs, J. London Math. Soc. (2) 10 (1975), 507-512.

5. A. Gardiner, An elementary classification of distance-transitive graphs of valency four, Ars Combin., to appear.

6. A. Gardiner, Antipodal covering graphs, J. Combinatorial Theory B 16 (1974), 255-273.

7. A. Gardiner, Arc transitivity in graphs III, Quart. J. Math. Oxford (2) 27 (1976), 313-323.

8. A. J. Hoffman and R. R. Singleton, On Moore graphs of diameters 2 and 3, IBM J. Res. Develop. 4 (1960), 497-504.

9. B. HUPPERT, Endliche Gruppen (Springer, 1967).

10. A. A. Ivanov, A. V. Ivanov and I. A. Faradżev, Distance-transitive graphs of valency 5, 6 and 7, Zh. Vychisl. Mat. i Mat. Fiz. 24 (1984), 1704-1718 (in Russian).

11. D. H. SMIтH, On tetravalent graphs, J. London Math. Soc. (2) 6 (1973), 659-662.

12. D. H. Sмiтh, Distance-transitive graphs of valency four, J. London Math. Soc. (2) 8 (1974), $377-384$.

13. D. H. Sмгтн, On bipartite tetravalent graphs, Discrete Math. 10 (1974), 167-172.

14. R. WeIss, Distance-transitive graphs and generalised polygons, Arch. Math., to appear.

15. H. Wielandt, Permutation Groups (Academic Press, 1964).

DEPARTMENT OF MATHEMATICS

UNIVERSITY OF BIRMINGHAM

BirminghaM B15 2TT, U.K.
DEPARTMENT OF MATHEMATICS

UNIVERSITY OF WESTERN AUSTRALIA

Nedlands, WA 6009, Australia 